\title{
Manufacture of chhana podo by incorporation of passion fruit (Passiflora edulis Sims fo. edulis) pulp
}

\author{
Dhanya Suresh, SN Rajakumar and Krupa Joseph
}

Received: 04 May 2019 / Accepted: 23 July 2019 / Published online: 28 October 2019

(C) Indian Dairy Association (India) 2019

\begin{abstract}
A study on manufacture of chhana podo prepared by using cow milk by incorporation of passion fruit (Passiflora edulis Sims fo. edulis) pulp was carried out. The attempts have been made to study effect of different levels of passion fruit pulp, 0 (T1), 32\% (T2), 34\% (T3), 36\% (T4) and 38\% (T5) on sensory quality of chhana podo. Based on the sensory analysis, chhana podo prepared with $32 \%$ passion fruit pulp (T2) was found superior over rest of the treatments. The chemical, microbial quality and color characteristics of both control and optimized treated sample were evaluated in the study. The latter was also estimated for total polyphenol content and was found to be 151.1 $\mathrm{mg} / 100 \mathrm{~g}$.
\end{abstract}

Keywords: Chhana podo, Chemical quality, Passion fruit pulp, Polyphenol content

\section{Introduction}

Chhana podo is the only baked indigenous dairy product which is prepared from chhana (Kadam et al. 2017). According to the revised standards of FSSAI (2006), Chhana or Paneer means the

College of Dairy Science and Technology, Kerala Veterinary and Animal Sciences University, Mannuthy, Thrissur, Kerala, India

SN Rajakumar $(\bowtie)$

Dairy Technology Department,

College of Dairy Science and Technology, Kerala Veterinary and Animal Sciences University, Mannuthy, Thrissur, Kerala, India

E-mail: rajakumar@kvasu.ac.in, Mobile: +919495882953 product obtained from any variant of milk, with or without added milk solids, by precipitation with permitted acidulants and heating. The process parameters for production of chhana from low fat cow milk were optimized by Jonkman \& Das in 1993. In India, annual production of Chhana is about 1,00,000 tons (Chawla et al. 2009). Chhana podo, the sweet chhana based delicacy is a unique product of east India which is characterized by light brown color with sweet caramelized flavor and rich taste. It has moist crumb, moderately spongy cake-like texture and delicate body. Chhana podo has no proper size and shape and it is prepared as per desiredness. The size, shape and appearance of the product vary depending upon the manufacturers. Ghosh et al. (2002) conducted a market survey of chhana podo sold in Orissa with its characterization and utilization.

The traditional method of its production involves mixing of ingredients such as chhana, sugar, suji or maida at ambient temperature and prolonged baking in a microwave/ convection oven. Baking is the key step in the preparation of Chhana Podo. It is a complex process in which physical and biochemical changes occur simultaneously. Dash et al. (1999) reported a method for the preparation of Chhana Podo by mixing Chhana, sugar and refined wheat flour/semolina in the ratio of 20:5:1 (w/w) with small quantities of heated and clarified butter oil (ghee), and by baking at a temperature-time combination of $150^{\circ} \mathrm{C}$ for $90 \mathrm{~min}$.

Kumar et al. (2002) reported on the optimization of ingredient and processing parameters during the manufacture of Chhana podo. Milk standardized to $4.5 \%$ fat and $8.5 \%$ SNF was heated to $85^{\circ} \mathrm{C}$ for $10 \mathrm{~min}$. Citric acid solution was added for coagulation of milk. Coagulum was retained in whey for $15 \mathrm{~min}$ followed by dewatering using a specially designed centrifugal whey separation system. Then chhana was mixed with specified amount of 5\% suji, 35\% sugar and water in dough mixing system. The prepared Chhana podo premix was transferred to a rectangular aluminium pan previously lined with melted ghee. The covered pan was transferred to a hot air oven maintained at $200 \pm 5^{\circ} \mathrm{C}$, and was kept for $50 \mathrm{~min}$.

Sensory and textural attributes showed many variations with regard to body and texture, color and appearance, flavor, sweetness and overall acceptability when observed in market 
sample of Chhana podo which was collected from reputed sweets shops of Bhubaneshwar. It was observed that most preferred varieties having pleasant, and caramel flavor, fully developed coherent pores with moderate degree of softness, outer surface light brown and centre portion white, moist surface and optimum sweetness (Kumar, 2008).

Passion fruit is a popular name given to several species of the genus Passiflora that belongs to Passifloraceae family, which is distributed in regions of tropical and subtropical climate of the world. These fruits are important for their pulp, peel and possess high levels of bioactive compounds (Reis et al. 2018). Passion fruit appears to be a rich source of nutrients such as carbohydrates, vitamins, and minerals that are essential for life. The fruit contains large amount of nutraceutical, such as phenolic acids, in which anthocyanins and flavonoids are the majoritarian compounds. Espírito-Santo et al. (2013) studied the possibility of using passion fruit fiber as a neutral ingredient for the design of new high value-added yoghurt and also reported the rheology, spontaneous whey separation, microstructure and sensorial characteristics of probiotic yoghurts enriched with passion fruit fiber. Studies have been conducted in using passion fruit as a raw material for the development of juice beverage with rich flavor and pleasant taste (Zhu et al. 2017).

The antioxidant activity of passion fruit dietary fibre has been studied using ethanol solvent with ABTS (2,2'-azino-bis(3ethylbenzothiazoline-6-sulphonic acid), DPPH (2,2-diphenyl-1picrylhydrazyl) and FRAP (Ferric Reducing Antioxidant Power) assay and was determined to be $2.1 \mu \mathrm{M}$ TE (Trolox Equivalent )/ g, $1.5 \mu \mathrm{M} \mathrm{TE} / \mathrm{g}$ and 4.6 $\mu \mathrm{M} \mathrm{TE} / \mathrm{g}$ respectively (Martínez et al. (2012). It has been discovered that piceatannol $(3,4,30,50$ tetrahydroxytrans-stilbene) is present in passion fruit seeds in large amounts and that this compound is the major component responsible for the passion fruit seed extract effects observed on melanogenesis and collagen synthesis (Matsui et al. 2010).

Kishore et al. (2011) evaluated the Total Soluble Solid content and titrable acidity in purple passion fruit pulp Passiflora edulis Sims which were found to be 15.30 and $3.80 \%$ respectively and total carotenoid content as determined by Pongener et al. (2013) was found to be $1467 \mu \mathrm{g} / 100 \mathrm{ml}$. Chemical characterization of passion fruit Passiflora edulis $f$. flavicarpa seeds have been done by Silva et al. (2015) and found to have high nutritional value, proving to be a promising product, mainly because it contains significant amounts of proteinase. Mamede et al. (2017) studied the volatile composition of sweet passion fruit (Passiflora alata Curtis) and revealed the main classes as esters and terpenes. The aim of this study was to evaluate the chemical characteristics, color parameters and total polyphenol content of optimized treated sample (T2) of chhana podo. Excellent carotenoid profile and the presence of phenolic compounds such as carotenoids, flavonoids, anthocyanins and provitamin A content, of passion fruit extract is expected to confer bioactive and therapeutic properties to the product.

\section{Materials and Methods}

\section{Manufacture of chhana podo}

For the preparation of chhana podo, cow milk was received from Dairy plant, College of Dairy Science and technology, Mannuthy, Thrissur, whereas passion fruit, baking powder, suji, and sugar were purchased from the local market. The chhana podo was prepared using milk of 3.5 fat and 8.7 SNF as per the procedure given below. Passion fruit pulp used for the preparation of treated samples possessed acidity of $2.765 \%$ citric acid. Some preliminary trials were conducted to determine appropriate level of addition of passion fruit pulp for incorporation in chhana podo. Four levels of addition of passion fruit pulp $(32,34,36$ and $38 \%)$ were selected on the basis of preliminary trials for further studies in six replications.

\section{Sensory evaluation}

In this study the organoleptic quality of control and treated samples of chhana podo was evaluated at by a panel of five semi trained judges on a 9-point hedonic scale where a score of 5 point represented 'dislike extremely' and score of 9 point represented 'like extremely' (Amerine et al. 1965). The samples for evaluation were marked suitably before serving the samples to the judges for sensory evaluation. The samples were evaluated in a sensory evaluation room with appropriate facilities.

\section{Proximate analysis and total phenol content}

The fat content of milk and chhana podo was determined by using standard Gerber method as per IS: 1224 (part-I), 1977. The acidity of milk and chhana podo was estimated according to IS: 1479, (part-I), 1960. The total solids and protein content of chhana podo were determined as per IS: 1479 (part-II), 1961. The ash content of chhana podo was determined as per the procedure quoted by AOAC (1975). The acidity of passion fruit pulp was determined as per the procedure given in AOAC (1975).

Total phenol content of the optimized treated sample was estimated as per the procedure mentioned by Swain and Hillis (1959). Data were statistically analyzed according to Snedecor and Cochran (1994) using randomized block design.

\section{Microbial analysis}

The microbiological analysis of the product was carried out as per the method described in IS: 1224 (part-I and part-II), 1981. Yeast \& Mold Count was determined by plating 1:10 dilution of chhana podo suspension using Potato Dextrose Agar (Hi Media). The count was taken after 3- 5 days of incubation at $30-35^{\circ} \mathrm{C}$. Coliform count was determined by plating 1:10 dilution of the 


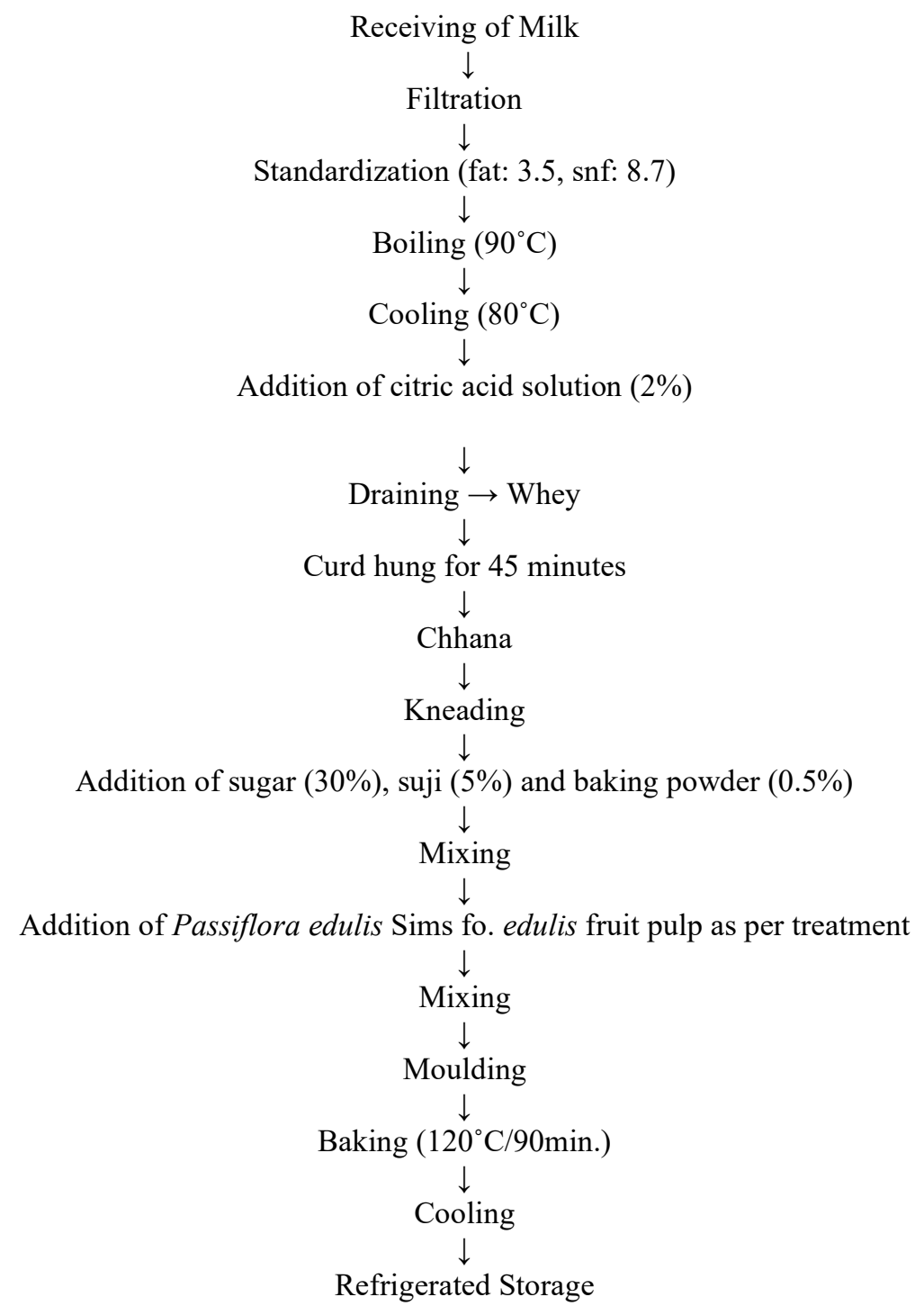

Fig.1a Optimized process of preparation of passion fruit pulp incorporated chhana podo

sample. Dilutions were plated using Violet Red Bile Agar (VRBA) media. These plates were incubated at $35^{\circ}-37^{\circ} \mathrm{C}$ for $24-48$ hours and there after counts were taken.

\section{Evaluation of color characteristics}

Color parameters of the samples of chhana podo were measured by MiniScan EZ spectrophotometer. Both control and optimized samples were tempered to room temperature and crushed using pestle and mortar. Crushed samples were filled in petriplates for taking the measurement. The color parameters such as ' $\mathrm{L}^{*}$ ', ' $\mathrm{a}$ ', and ' $\mathrm{b}$ ', values were noted; The color parameter ' $\mathrm{L}$ ' ' is a measure of lightness or luminance, which ranges from 0 to $100\left[\mathrm{~L}^{*}=0\right.$ (black) and $\mathrm{L}^{*}=100$ (white)] and ' $\mathrm{a}$ *' ' and ' $\mathrm{b}$ *' ' are the two chromatic components, which range from -120 to $120\left[\mathrm{a}^{*}=-120\right.$ (green) and $\mathrm{a} *=120($ red) $]$ and $\left[\mathrm{b}^{*}=-120\right.$ (blue) and $\mathrm{b}^{*}=120$ (yellow)] respectively.

\section{Statistical analysis}

Data obtained from various experiments during optimization, was statistically analyzed by using statistical software SPSS 21.0. One way ANOVA for sensory evaluation of samples of chhana podo was done using Tukey test of SPSS.

\section{Results and Discussion}

\section{Sensory evaluation}

Table 1 disports the average scores obtained for control and treated samples for different sensory attributes. Higher score for flavor was secured by the control, T1 $(7.82 \pm 0.28)$ than other 

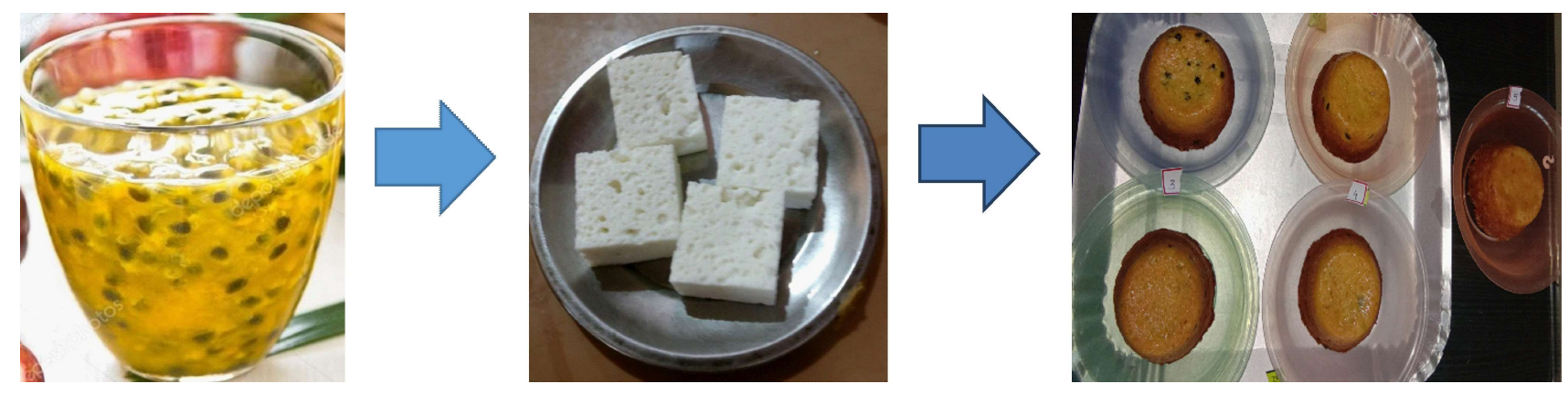

Fig.1b Steps involved in the preparation of passion fruit pulp incorporated chhana podo

Table 1 Effect of incorporation of passion fruit pulp on the sensory attributes of chhanapodo

\begin{tabular}{|c|c|c|c|c|c|}
\hline \multirow[b]{2}{*}{ Attributes } & \multicolumn{4}{|c|}{ Sensory Scores on 9 point hedonic scale } & \multirow[b]{2}{*}{ T5 } \\
\hline & $\mathrm{T} 1$ & $\mathrm{~T} 2$ & $\mathrm{~T} 3$ & $\mathrm{~T} 4$ & \\
\hline Flavor & $7.82 \pm 0.28^{\mathrm{c}}$ & $7.55 \pm 0.19^{\mathrm{bc}}$ & $6.83 \pm 0.17^{\mathrm{a}}$ & $7.05 \pm 0.42^{\mathrm{ab}}$ & $6.94 \pm 0.25^{\mathrm{a}}$ \\
\hline Color \& & $8.00 \pm 0.00^{c}$ & $7.77 \pm 0.09^{\mathrm{bc}}$ & $6.63 \pm 0.12^{\mathrm{a}}$ & $6.83 \pm 0.17^{\mathrm{a}}$ & $7.22 \pm 0.25^{\mathrm{b}}$ \\
\hline Appearance & & & & & \\
\hline Body \& Texture & $7.77 \pm 0.50^{\mathrm{b}}$ & $7.66 \pm 0.16^{b}$ & $7.10 \pm 0.17^{\mathrm{ab}}$ & $6.88 \pm 0.09^{\mathrm{a}}$ & $7.27 \pm 0.69^{\mathrm{ab}}$ \\
\hline $\begin{array}{l}\text { Overall } \\
\text { Acceptability }\end{array}$ & $8.83 \pm 0.86^{\mathrm{c}}$ & $7.66 \pm 0.16^{\mathrm{b}}$ & $6.88 \pm 0.34^{\mathrm{ab}}$ & $6.72 \pm 0.25^{\mathrm{a}}$ & $7.10 \pm 0.25^{\mathrm{ab}}$ \\
\hline
\end{tabular}

Note: Figures are mean \pm standard deviation of six replications. Values with different superscripts

in a row are significantly different $(\mathrm{p} \mathrm{d}$ " 0.01$)$

T1: Chhana podo without pulp; T2: Chhana podo with $32 \%$ pulp; T3: Chhana podo with $34 \%$ pulp; T4: Chhana podo with $36 \%$ pulp; T5: Chhana podo with $38 \%$ pulp

treatments \& this may be probably due to the innate caramelized cooked and fresh flavor possessed by the product. Chhana podo incorporated with $32 \%$ fruit pulp was found to be superior in terms of acceptability and flavor. The scores obtained by sample $\mathrm{T} 2$ for color and appearance $(7.77 \pm 0.09)$ was not significantly (p d" 0.01) different from that of the control sample (T1) and this may be due to the reason that the amount of pulp added (32\%) was not enough to make a noticeable change in the appearance of chhana podo. In the case of body and texture, control, T1 (7.77 $\pm 0.50)$ and chhana podo with $32 \%$ pulp, T2 (7.66 \pm 0.16$)$ samples scored the highest score compared to T3 and T4 samples. This can be accounted by the uniform baking and porosity possessed by the samples. The overall acceptability scores obtained by $\mathrm{T} 1$ $(8.83 \pm 0.86)$ and $\mathrm{T} 2(7.66 \pm 0.16)$ samples was found to be higher than the other treatments. From the overall scores obtained for sensory attributes, it can be concluded that the chhana podo sample incorporated with $32 \%$ passion fruit pulp (T2) has exhibited better acceptability among other treatments and also showed higher scores resembling to that of control chhana podo (T1).

\section{Proximate composition}

The proximate analysis of control and optimized sample (T1) of chhana podo prepared by 0 and $32 \%$ levels of passion fruit pulp was carried out for moisture, fat, protein,

ash and titratable acidity. The results are showed in Table 2 .

\section{Fat content}

The data from the study revealed that the variation in the fat content of chhana podo due to the incorporation of passion fruit pulp was found to be significant. The fat content decreased significantly with the addition of pulp. The average fat percentage of chhana podo at 0 and $32 \%$ pulp addition were 14.73 and 13.17 percent respectively. The obvious reason is that due to the very low fat content of passion fruit pulp, there was reduction in the amount of fat in the product. Secondly, due to very low fat content of passion fruit pulp (Adeyeye and Aremu, 2017), its addition reduced the fat of the final product.

The main source of fat in chhana podo was mainly from chhana which contained on an average 23.17 per cent fat. The higher fat content was observed at T1 ( 0 per cent) i.e. podo without pulp. These results are quite comparable with the result reported by Kumar et al. (2002) who reported 15.50 per cent fat. Mane (2006) found the average per cent fat as 15.06 in chhana podo.

\section{Moisture content}

The moisture content of chhana podo showed significant increase in sample incorporated with $32 \%$ passion fruit pulp compared to that of control sample. This was obviously due to 
Table 2 Chemical quality of control and optimized sample of passion fruit pulp incorporated chhana podo (\%)

\begin{tabular}{lllc}
\hline & \multicolumn{2}{l}{ Chemical composition } & Significance \\
\cline { 2 - 3 } & $\mathrm{T} 1$ & $\mathrm{~T} 2$ & $\mathrm{~S}^{*}$ \\
\hline Fat & $14.73 \pm 0.195$ & $13.17 \pm 0.239$ & $\mathrm{~S}^{*}$ \\
Moisture & $30.295+-0.522$ & $34.495+-0.315$ & $\mathrm{~S}^{*}$ \\
Acidity & $0.23 \pm 0.007$ & $0.64 \pm 0.023$ & $\mathrm{~S}^{*}$ \\
Ash & $1.65 \pm 0.034$ & $1.36 \pm 0.02$ & $\mathrm{~S}^{*}$ \\
Protein & $17.67 \pm 0.091$ & $18.85 \pm 0.132$ & \\
\hline
\end{tabular}

Note: $\mathbf{S} *$-Significant at $5 \%$ level of significance; $\mathbf{N S}{ }^{*}$ - Not significant at $5 \%$ level of significance

Table 3 Color parameters of control and optimized sample of passion fruit pulp incorporated chhana podo

\begin{tabular}{lllc}
\hline Parameters & $\mathrm{T} 1$ & $\mathrm{~T} 2$ & Significance \\
\hline $\mathrm{L}^{*}$ & $58.33 \pm 0.540$ & $56.55 \pm 0.306$ & $\mathrm{~S}^{*}$ \\
$\mathrm{a}^{*}$ & $10.67 \pm 0.115$ & $9.51 \pm 0.146$ & $\mathrm{~S}^{*}$ \\
$\mathrm{~b}^{*}$ & $28.24 \pm 0.36$ & $36.26 \pm 0.54$ & $\mathrm{~S}^{*}$ \\
\hline
\end{tabular}

Note: $\mathbf{S} *$ - Significant at $5 \%$ level of significance; $\mathbf{N S}$ - Not significant at $5 \%$ level of significance

very high moisture content of passion fruit pulp (seed and juice) (Adeyeye and Aremu, 2017). The chhana podo without passion fruit pulp contained 30.29 per cent moisture. The average values for moisture content in chhana podo were more or less similar to the figures reported by Kumar (2008) and Dash et al. (1999) as 65.57 to 73.29 per cent total solids. Mane (2006) observed an average of 74.81 per cent total solids in chhana podo.

\section{Acidity}

Addition of passion fruit pulp has resulted in significant increase in acidity of chhana podo. Acidity of control (T1) and optimized treated sample (T2) of chhana podo was found to be 0.23 and $0.64 \%$ lactic acid respectively. This increase in acidity was statistically significant $(p>0.05)$ which could be related to the increased acidity of passion fruit pulp $2.76 \%$ citric acid as compared to control chhana podo ( $0.23 \%$ lactic acid). Dash et al. (1999) reported that the titratable acidity of chhana podo samples laid in the range of 0.18 to 0.27 per cent and average value was found to be $0.20 \pm 0.2$ per cent. Theses variations in acidity were justified by the quality of raw milk and the amount and strength of coagulant used for chhana preparation.

Kumar et al. (2002) found 0.25 per cent acidity in chhana podo while optimizing the process for commercial production of chhana podo. The results of present study with respect to acidity were more or less in accordance with the findings reported by Dash et al. (1999) and Kumar et al. (2002). The results showed in Table 2 clearly reveal that the acidity of chhana podo increased significantly with the addition of passion fruit pulp.

\section{Ash content}

The average ash content of control and optimized treated sample of chhana podo was 1.65 and 1.36 per cent, respectively. The results for ash content depicted that addition of passion fruit pulp resulted in decrease in the ash content of chhana podo. This may be probably due to very low ash content of passion fruit juice (0.34\%) (Adeyeye and Aremu, 2017).

Ghosh et al. (1998) observed that the ash content of chhana podo samples lay in the range of 0.8-1.0 per cent. Dash et al. (1999) reported that the ash content of chhana podo samples were in the range of 0.93 to 1.52 per cent. The values obtained in the present study showed similar results. Dash et al. (1999) noted that the major source of ash in chhana podo was contributed by chhana and it changed with the initial quality of raw milk used for preparation.

\section{Protein content}

The protein content in the optimized treated sample of chhana podo (T2) was found to be significantly different from that of control sample (T1). An increase in protein content was noted in the treated sample due to the addition of pulp which contained protein as about $6.53 \mathrm{~g} / 100 \mathrm{~g}$ of dry weight (Reis et al, 2018). Control and treated samples of chhana podo contained 17.67 and $18.85 \%$ protein content respectively.

Protein content in chhana podo was studied by Ghosh et al. (1998), Dash et al. (1999), Kumar et al. (2002), and Mane (2006)., and was found to be 15 to $17,16.3$ to $21.08,12$ and 13.90 per cent respectively. These values are in agreement with the values obtained in the present study. Chhana was the major contributor of protein in chhana podo (Dash et al. 1999) and it was variable depending on the initial composition of milk used.

\section{Total polyphenol content}

Passion fruit is considered to have excellent functional properties due to the presence of its various nutritional components like phenolic constituents, anthocyanin, flavonoids, carotenoids and 
other bioactive compounds. The amount of these nutritional elements was influenced by factors such as genotype, geographic effect, crop year, maturation and storage conditions (Souza et al. 2008; Carden osa et al. 2016). Li et al. (2016) have summarized about antioxidant, anti-hypertension, anti-fungus, anti-diabetes, cardiovascular protection properties and anti-inflammation activities of passion fruit in their literature.

Total polyphenol content of the optimized treated sample was determined and was found to be $151.1 \mathrm{mg} / 100 \mathrm{~g}$. Amount of total carotenoids and phenolic compounds in passion fruit (Passiflora edulis Sims fo. edulis) pulp was reported to be $288.56 \mu \mathrm{g}$ and $788.93 \mathrm{mg}$ per $100 \mathrm{~g}$ dry weight respectively (Reis et al. 2018). They also found out the total flavonoid content in pulp to be $229.79 \mathrm{mg} / 100 \mathrm{~g}$ and about $12.35 \mathrm{mg} / 100 \mathrm{~g}$ dry weight kaempherol, the flavonoid constituent. These results revealed that passion fruit pulp have the potential to be used in different food formulations that would enhance its beneficial properties due to the presence of these nutritional components.

\section{Microbiological quality}

The coliform count in both control and optimized treated samples were nil whereas the yeast and mold count were found to be 30 and $50 \mathrm{cfu} / \mathrm{g}$ respectively being higher than that of reported by Kumar (2008) but within the limits of FSSAI microbiological standards, 2015. Higher yeast and mold count found in the treated samples may be due to the higher moisture content as a result of passion fruit pulp addition.

\section{Color characteristics}

The lightness value (L*) of control (T1) and optimized sample (T2) was found to be 58.33 and 56.55 respectively and from this result, it can be inferred that the addition of pulp resulted in a decrease in lightness value of chhana podo. The values of chromatic components of control sample were found to be 10.67 and 28.24 respectively while those of optimized sample were 9.51 and 36.26. The values of $\mathrm{L}^{*}$ and $\mathrm{a}^{*}$ of T1 and T2 samples were significantly different. Kumari (2013) evaluated the lightness value of the upper crust of chhana podo and the value was found to be decreasing with increasing temperature and time during baking.

\section{Conclusions}

From the present study, it can be concluded that the addition of passion fruit pulp has increased the functionality of chhana podo and this product had the potential to gain flavour among consumers due to its therapeutic and nutritive advantages. From the sensory analysis of the treatment samples, higher acceptability was realized for $\mathrm{T} 2$ sample of chhana podo (with $32 \%$ passion fruit pulp added) than remaining treatments. The chemical composition of the control and optimized sample was studied and total polyphenol content of the latter has also been estimated which evidenced its significant beneficial effects on health.

\section{Acknowledgements}

The first author gratefully acknowledges the financial assistance received from CDST, Mannuthy (Kerala veterinary and animal sciences University), Kerala, India in the form of Institutional Fellowship for carrying out the present project as well as to the CARe KERALAM Ltd, Koratty, Kerala for the technical support rendered towards estimation of Total Polyphenol content.

\section{References}

Adeyeye EI, Aremu MO (2017) Chemical composition of the raw fruit coat, seed and pulp of passion fruit (Passiflora edulis). Trends Sci Technol J 2: 334-341

Amerine MA, Panborn EB, Roessler EB (1965) Principles of sensory evaluation of food. Academic Press, New York

Aneja RP, Banerjee AK, Chandan RC, Mathur BN (2002) Technology of Indian Milk products. Dairy India publication. Delhi: 4-22

AOAC (1975) Official methods of analysis. Association of Official Analytical Chemists, Washington, D.C, U.S.A. 16th Edition

BIS: 1224 (1977) Determination of Fat by Gerber Method- Milk Products. Bureau of Indian Standards, New Delhi, (Part II)

BIS (1981) (SP: 18). Handbook of Food Analysis. Part XI., Dairy Products. Bureau of Indian Standards, New Delhi: 340.

BIS: Methods for test for dairy industry. Rapid examination of milk. Indian Standards Institution, Manak Bhavan, New Delhi, 1479-1960, (Part -I)

BIS: Methods of test for dairy industry. Chemical analysis of milk. Indian Standards Institution, Manak Bhavan, New Delhi, India, 1479-1961, (Part - II)

Cardeñosa V, Vilaplana GA, Muriel JL, Moreno DA, MorenoRojas JM (2016) Influence of genotype, cultivation system and irrigation regime on antioxidant capacity and selected phenolics of blueberries (Vaccinium corymbosum L.). Food Chem 202: 276-283

Chawla A, Chawla N, Pant Y, Kandhari P (2009) Milk and dairy products in India-production, consumption and exports. Hindustan Studies and Services Limited. Bhopal. India.

Dadali G, Demirhan E, Ozbek B (2007) Color change kinetics of spinach undergoing microwave drying. Drying Technol 25: 1713-1723

Dash DK, Ghatak PK, Das A (1999) Laboratory made chhanapodo. J Dairying. Foods Home Sci 18:127-129

Espírito-Santo AP, Lagazzo A, Sousa ALOP, Perego P, Converti A, Oliveira MN (2013) Rheology, spontaneous whey separation, microstructure and sensorial characteristics of probiotic yoghurts enriched with passion fruit fiber. Food Res Int 50: 224-231

Ghosh BC, Rao JK, Balasubramanyam BV, Kulkarni S (2002) Market survey of chhana poda sold in Orissa, its characterisation and utilisation. Indian Dairyman 54: 37- 41

Jonkman MJ, Das H (1993) Optimization of process parameters for production of chhana from low fat cow milk. J Food Sci Technol 30: 417-421

Kishore K, Pathak KA, Shukla R, Bharali R (2011) Effect of storage temperature on physico-chemical and sensory attributes of purple passion fruit (Passiflora edulis Sims). J Food Sci Technol 48: 484488

Kumar R (2008) Technology of dietetic chhana podo production. Thesis, National Dairy Research Institute. Karnal. 99pp 
Kumar S, Khuamrui K, Bandyopadhyay P (2002) Process optimization for commercial production of Chhana podo. Indian Dairyman 54: 61

Kumari A (2013) Evaluation of physico-thermal and textural properties of chhana podo and optimization of its baking process using response surface methodology. M. Tech thesis, National Dairy Research Institute, Bangalore. 86pp

Li H, Gao R, Zheng J, Zhang J, Zhang D, Dong Z, Zhou T, Li H (2016) Bioactivities of Passion Fruit. Int J Traditional Natural Med 6: 2634

Londhe SV, Ambadkar RK, Patil DP (2017) Suitability of buffalo milk for prepration of chhana podo. Life Sci Int Res J 4

Mamede MGNA, Soares AG, Oliveira EG, Farah A (2017) Volatile Composition of Sweet Passion Fruit (Passiflora alata Curtis). J Chemistry

Mane JR (2006) Studies on manufacture of chhana podo. Thesis submitted to Dr. Balasaheb Sawant Konkan Krishi Vidyapeeth, Dapoli

Martínez R, Torres P, Meneses MA, Figueroa JG, Pérez-Álvarez JA, ViudaMartos M (2012) Chemical, technological and in vitro antioxidant properties of mango, guava, pineapple and passion fruit dietary fibre concentrate. Food Chem 135: 1520-1526

Matsui Y, Sugiyama K, Kamei M, Takahashi T, Suzuki T, Katagata Y, Ito $\mathrm{T}$ (2010) Extract of Passion Fruit (Passiflora edulis) Seed Containing High Amounts of Piceatannol Inhibits Melanogenesis and Promotes Collagen Synthesis. J. Agric. Food Chem 58: 11112-11118

Mukhopadhyay S,Majumdar GC, Goswami TK, Mishra HN (2013) Fuzzy logic (similarity analysis) approach for sensory evaluation of chhana podo. LWT- Food Sci Technol 53: 204-210

Mukhopadhyay S (2012) Optimization of process parameters for the production of chhana podo. MS dissertation. Indian Institute of Technology. Kharagpur. West Bengal. India
Ovando CA, Pacheco-Herna'ndez ML, Pa'ez-Herna'nde ME, Rodrý'guez JA, Gala'n-Vidal CA (2009) Review: chemical studies of anthocyanins. Food Chem 113: 859-871

Patel HG, Upadhyay KG (2003) Standardization of compositional recipe of chhana podo - level of sugar addition. J Food Sci Technol 40: 8992

Pongener A, Sagar V, Pal RK, Asrey R, Sharma RR, Singh SK (2013) Physiological and quality changes during postharvest ripening of purple passion fruit (Passiflora edulis Sims). J Fruits 69: 19-30

Rao KJ, Dhas PHA, Emerald FME, Ghosh BC, Balasubramanyam BV, Kulkarni S (2006) Moisture sorption characteristics of chhana podo at $5^{\circ} \mathrm{C}$ and $35^{\circ} \mathrm{C}$. $\mathrm{J}$ Food Eng 76: 453-459

Reis LCR, Facco EMP, Salvador M, Hickmann S, Rios AO (2018) Antioxidant potential and physicochemical characterization of yellow, purple and orange passion fruit. J Food Sci Technol 55: 2679-2691

Sawant ST, Jayashri U, Pisal, Desale RJ, Kadam DG (2010) Study on sensory qualities of chhana podo from cow milk. J Dairying Foods HS 29: 193-19

Silva RM, Placido GR, Silva MAP, Castro CFS, Lima MS, Caliari M (2015) Chemical characterization of passion fruit (Passiflora edulis $\mathrm{f}$. flavicarpa) seeds. African J Biotech 14: 1230-1233

Snedecor WG, Cochran GW (1994) Statistical methods, East West Press Pvt. Ltd, New Delhi

Souza LM, Ferreira KS, Chaves JBP, Teixeira SL (2008) L-ascorbic acid, $\mathrm{b}$-carotene and lycopene content in papaya fruits (carica papaya) with or without physiological skin freckles. Sci Agric 65: 246-250

Swain T, Hillis WE (1959) The phenolic constituents of Prunus domestica I. The quantitative analysis of phenolic constituents. J Sci Food Agric 10: 63-68

Zhu X, Duan Z, Yang Y, Huang X, Xu C, Huang Z (2017) Development of passion fruit juice beverage. Earth Environ Sci 100 